\title{
First Results of the Phase II SIMPLE Dark Matter Search
}

\author{
M. Felizardo, ${ }^{1,2,3}$ T. Morlat, ${ }^{3,4}$ A.C. Fernandes, ${ }^{2,3}$ TA Girard,, 3, , J.G. Marques, ${ }^{2,3}$ A.R. Ramos, ${ }^{2,3}$ M. \\ Auguste,${ }^{5}$ D. Boyer ${ }^{5}$ A. Cavaillou,${ }^{5}$ C. Sudre,${ }^{5}$ J. Poupeney, ${ }^{5}$ R.F. Payne, ${ }^{6}$ H.S. Miley,${ }^{6}$ and J. Puibasset ${ }^{7}$ \\ (The SIMPLE collaboration) \\ ${ }^{1}$ Department of Physics, Universidade Nova de Lisboa, 2829-516 Caparica, Portugal \\ ${ }^{2}$ Instituto Tecnológico e Nuclear, Estrada Nacional 10, 2686-953 Sacavém, Portugal \\ ${ }^{3}$ Centro de Física Nuclear, Universidade de Lisboa, 1649-003 Lisbon, Portugal \\ ${ }^{4}$ Department of Physics, Universidade de Lisboa, Campo Grande C8, 1749-016 Lisboa, Portugal \\ ${ }^{5}$ Laboratoire Souterrain à Bas Bruit, Observatoire de la Côte d'Azur, 84400 Rustrel-Pays d'Apt, France \\ ${ }^{6}$ Pacific Northwest National Laboratory, Richland, WA 99352 USA \\ ${ }^{7}$ Centre de Recherche sur la Matiére Divisée CNRS et Université d'Orléans, 45071 Orléans, cedex 02 France
}

(Dated: October 30, 2018)

\begin{abstract}
We report results of a $14.1 \mathrm{kgd}$ measurement with 15 superheated droplet detectors of total active mass $0.208 \mathrm{~kg}$, comprising the first stage of a $30 \mathrm{kgd}$ Phase II experiment. In combination with the results of the neutron-spin sensitive XENON10 experiment, these results yield a limit of $\left|a_{p}\right|<0.32$ for $\mathrm{M}_{W}=50 \mathrm{GeV} / \mathrm{c}^{2}$ on the spin-dependent sector of weakly interacting massive particle-nucleus interactions with a $50 \%$ reduction in the previously allowed region of the phase space formerly defined by XENON, KIMS and PICASSO. In the spin-independent sector, a limit of $2.3 \times 10^{-5} \mathrm{pb}$ at $\mathrm{M}_{W}=45 \mathrm{GeV} / \mathrm{c}^{2}$ is obtained.
\end{abstract}

The direct search for weakly interacting massive particle (WIMP) dark matter continues to be among the forefront endeavors of modern physics activity. Search experiments are generically based on the detection of nuclear recoil events resulting from WIMP-nucleus interactions, and are traditionally classified as spin-independent (SI) or spin-dependent (SD) according to which interaction channel the experiment is most sensitive, of which the first has generally attracted the most attention. The current status of the SI search for WIMPs is defined by a number of projects, including XENON 1], CDMS [2] and ZEPLIN [3], which as a result of their target nuclei spins also define the WIMP-neutron sector of the SD phase space. The WIMP-proton sector is currently constrained by PICASSO [4] and KIMS [5].

The SIMPLE (Superheated Instrument for Massive ParticLe Experiments) [6, 7] project, located in a $61 \mathrm{~m}^{3}$ cavern at the 1500 mwe level of the Laboratoire Souterrain à Bas Bruit (LSBB) in southern France, currently runs superheated droplet detectors (SDDs). The SDD is a suspension of $1-2 \%$ superheated liquid $\mathrm{C}_{2} \mathrm{ClF}_{5}$ droplets $(\sim 30 \mu \mathrm{m}$ radius $)$ in a viscoelastic $900 \mathrm{ml}$ gel matrix which undergo transitions to the gas phase upon energy deposition by incident radiation. Two conditions are required for the nucleation of the gas phase in the superheated liquid [9]: (i) the energy deposited must be greater than a thermodynamically-defined minimum energy, and (ii) this energy must be deposited within a thermodynamically-defined maximum distance inside the droplet. Together, energy depositions of order $\sim$ $150 \mathrm{keV} / \mu \mathrm{m}$ are required for a bubble nucleation, which renders the SDD effectively insensitive to the majority of traditional detector backgrounds which complicate

\footnotetext{
*corresponding author criodets@cii.fc.ul.pt
}

more conventional dark matter search detectors (including electrons, $\gamma$ 's and cosmic muons). The insensitivity is not trivial, comprising an intrinsic rejection factor superior to that of other search techniques by 1-5 orders of magnitude. Additional advantages of the superheated technique include low cost, scalability, and increased sensitivity to WIMP-proton spin interactions via the ${ }^{19} \mathrm{~F}$ content [10].

The SDDs were fabricated according to previouslydescribed procedures [6], in an underground (210 mwe) clean room in close proximity to the measurement site. The SIMPLE gel ingredients, all biologically-clean food products, are purified using actinide-specific ionexchanging resins. The freon is single distilled; the water, double distilled. The presence of $\mathrm{U} / \mathrm{Th}$ contaminations in the gel, measured at $\sim 0.1 \mathrm{ppb}$ by low-level $\alpha$ and $\gamma$ spectroscopy of the production gel, yields an overall $\alpha$ background level of $<0.5 \mathrm{evt} / \mathrm{kg}$ freon $/ \mathrm{d}$. A similar level is measured for the detector containment materials.

The detectors are capped using a mechanical construction which virtually eliminates pressure microleaks [7]. Each cap contains feedthroughs for pressure monitoring, and for a $20-16 \mathrm{k} \mathrm{Hz}$ electret microphone encased in a latex sheath, which is immersed in a $4 \mathrm{~cm}$ thick glycerin layer covering the gel at the top of the detector containment. Each microphone response is preamplified and recorded in a MatLab platform in sequential files of $8 \mathrm{~min}$ duration 8], with resolutions of 0.3 $\mathrm{mV}$ in amplitude and $1.6 \times 10^{-2} \mathrm{~ms}$ in time; the pressure reading is similarly recorded separately. The use of shielded telecommunications-grade cabling eliminates signal resulting from cable motion and parasitics, even when exaggerated.

The SDDs are immersed to a depth of $20 \mathrm{~cm}$ in a 700 liter water pool maintained at a bath temperature of $9.0 \pm 0.1^{\circ} \mathrm{C}$ within the cavern, and pressurized to 2 bar to 
reduce background sensitivity. The water pool rests on a dual vibration absorber placed atop a $20 \mathrm{~cm}$ thick wood platform resting on a $50 \mathrm{~cm}$ thick concrete floor. The pool is surrounded by three layers of sound and thermal insulation. An additional 50-75 cm thick water shielding surrounds the insulated pool and pedestal, with a $75 \mathrm{~cm}$ water thickness overhead; $50 \mathrm{~cm}$ of water separates the pool bottom from the SDD bases.

At 1500 mwe, the ambient neutron flux is primarily due to the surrounding calcite rock, estimated at well-below $4 \times 10^{-5} \mathrm{n} / \mathrm{cm}^{2} \mathrm{~s}[11$. The cavern is shielded from the rock environment by a $30-100 \mathrm{~cm}$ thickness of concrete, internally sheathed by a $1 \mathrm{~cm}$ thickness of iron. Radioassays of the concrete yielded $1.90 \pm 0.05 \mathrm{ppm}{ }^{232} \mathrm{Th}$ and $0.850 \pm 0.081 \mathrm{ppm}{ }^{238} \mathrm{U}$; of the steel, $3.20 \pm 0.25 \mathrm{ppb}^{232} \mathrm{Th}$ and $2.9 \pm 0.2 \mathrm{ppb}{ }^{238} \mathrm{U}$. The results are at the same level as those recorded in other underground locations such as Canfranc, Modane and Gran Sasso 12 14. Monte-Carlo simulations of the on-detector neutron field, which include all shielding materials and account for spontaneous fission plus decay-induced $(\alpha, \mathrm{n})$ reactions, show negligible variations for concrete thicknesses $\geq 20 \mathrm{~cm}$, and yield an expected neutron background of $1.09 \pm 0.02$ (stat) \pm 0.07 (syst) evt/kgd.

The ambient radon level varies seasonally between 28 $1000 \mathrm{~Bq} / \mathrm{m}^{3}$ as a result of water circulation in the mountain. The cavern air is purged $\sim 10$ times per day, reducing the ambient radon levels to $\sim 60 \mathrm{~Bq} / \mathrm{m}^{3}$. Diffusion of the environmental radon into a detector is limited by the surrounding water, which covered the detectors to just above their glycerin levels, and is circulated at 25 liter/min (equivalent to replacing the top $1 \mathrm{~cm}$ water layer each minute). The radon contribution is also low because of the short radon diffusion lengths of the SDD construction materials (glass, plastic, metal), the $\mathrm{N}_{2}$ overpressuring which inhibits the advective influx of $\mathrm{Rn}$ (via stiffening of the gel), and the glycerin layer covering the gel. The reduction in the overall radon contribution to the measurement, including its progeny, is estimated at $\sim 10^{5}$ with the overall $\alpha$ contribution to the measurement (including the detector contribution) estimated at $3.26 \pm 0.08$ (stat) \pm 0.76 (syst) evt $/ \mathrm{kgd}$.

Data obtained from 15 SDDs, containing between 8-21 $\mathrm{g}$ of $\mathrm{C}_{2} \mathrm{ClF}_{5}$ for a total active mass of $0.208 \mathrm{~kg}$, between 27 October 2009 and 05 February 2010 were analyzed for this report. An additional, similarly installed, freon-less but otherwise identical SDD, served as an acoustic veto. The total raw exposure was $14.10 \pm 0.01 \mathrm{kgd}$, with 1.94 kgd resulting from the detectors being introduced at one device per day over the three week installation period, and a $4.70 \mathrm{kgd}$ loss from weather-induced power failures during the run.

The SDD signals, pressures and temperature are monitored continuously during operation, as also the radon level. Each detector was first inspected for raw signal rate and pressure evolution over the measurement period. An initial data set (4056 events) was then formed by passing the data files through a pulse validation routine 8] which tagged signal events if their amplitude exceeded the noise level of the detector by $2 \mathrm{mV}$. Tagged signals in coincidence with the freon-less device were next rejected, as also all candidate signals with less than five pulse spikes above threshold; the remaining set was then cross-correlated in time between all SDDs, and coincidences rejected as local noise events and that a WIMP interacts with no more than one of the in-bath detectors.

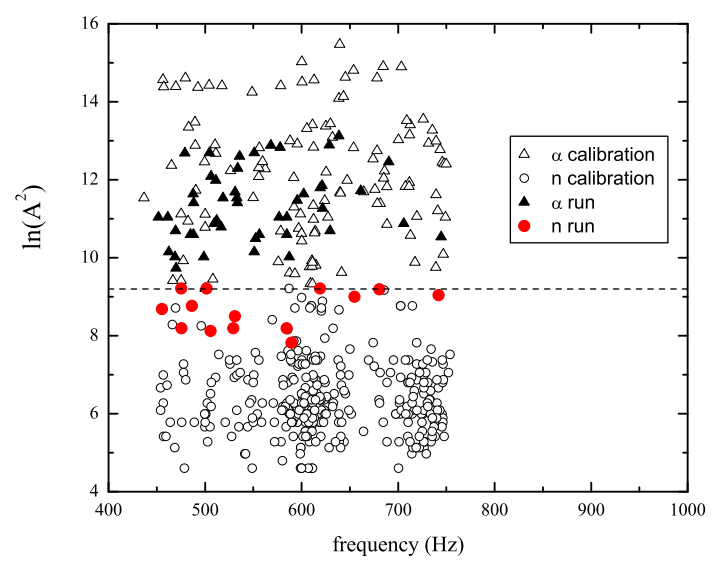

FIG. 1: Scatter plot of the squared amplitude and frequency of the primary harmonic of each true nucleation event (solid), together with the same for neutron and $\alpha$ calibration events (open), with the dashed horizontal line corresponding to a signal amplitude of $100 \mathrm{mV}$.

The signal waveform, decay time constant and spectral density structure of the remaining 1828 single events were next inspected individually. A particle-induced nucleation event possesses a characteristic frequency response, with a time span of a few milliseconds, a decay constant of 5-40 ms, and a primary harmonic between 0.45-0.75 $\mathrm{kHz}$ [8]. This response differs significantly from those of gel-associated acoustic backgrounds such as trapped $\mathrm{N}_{2}$ gas $(3.4 \%$ of the total), gas escape $(0.008 \%)$, and gel fractures $(4.4 \%)$ which appear at lower frequencies [8], as well as local acoustic backgrounds (88\%) such as water bubbles which differ in power spectra. This event-by-event analysis permits isolation of the true nucleation events with an efficiency of better than $97 \%$ at $95 \%$ C.L. Fig. 1 displays the signal amplitudes (A) with frequency for each of the identified 60 particle-induced signal events, in which a gap corresponding to $\mathrm{A}=100-130 \mathrm{mV}$ is discernible.

At $9^{\circ} \mathrm{C}$, the reduced superheat of the devices is 0.3 , and the probability of events from electrons, $\gamma$ 's and mip's negligible [6] over the exposure. Calibrations of the $\alpha$ response have been made by doping the devices with U308 during fabrication. The event signals, identified in the same fashion as described above, are shown in Fig. 1. The SRIM-calculated $\mathrm{dE} / \mathrm{dx}$ for $\alpha$ 's in $\mathrm{C}_{2} \mathrm{ClF}_{5}$ has a Bragg peak which sets a lower energy threshold at 200 $\mathrm{keV}$ for the operating temperature and pressure; below 
this threshold, $\alpha$ 's are detected only through $\alpha$-induced nuclear recoils.

Calibration of the high concentration SDD response to neutrons, using sources of $\mathrm{Am} / \mathrm{Be}$, yielded a minimum threshold recoil energy $\left(\mathrm{E}_{t h r}\right)$ of $8.0 \pm 0.1 \mathrm{keV}$, with an acoustic detection efficiency of $0.98 \pm 0.03$ at $9^{\circ} \mathrm{C}$ and 2 bar. The events are also displayed in Fig. 1, all of which occur with amplitudes $\leq 100 \mathrm{mV}$. The difference in the two distributions, particularly at lower amplitudes, results from performing the calibrations with a $15 \mathrm{~cm}$ water shield to enhance the tails on the moderated neutron spectrum.

Fig. 2 displays a typical histogram of both calibration signal amplitudes. As seen, the neutron-induced events are of lower amplitude than the $\alpha$-induced, and empirically well fit by a Gaussian plus constant background from which a discrimination cut for $\mathrm{A} \leq 100 \mathrm{mV}$ is placed with an acceptance of $>97 \%$. The small droplet size provides a natural lower cutoff to the deposited $\alpha$ energy and is together with the $\mathrm{dE} / \mathrm{dx}$ responsible both for the amplitude gap between the $\alpha$ and neutron populations, and the spectral asymmetry in the $\alpha$ distribution.

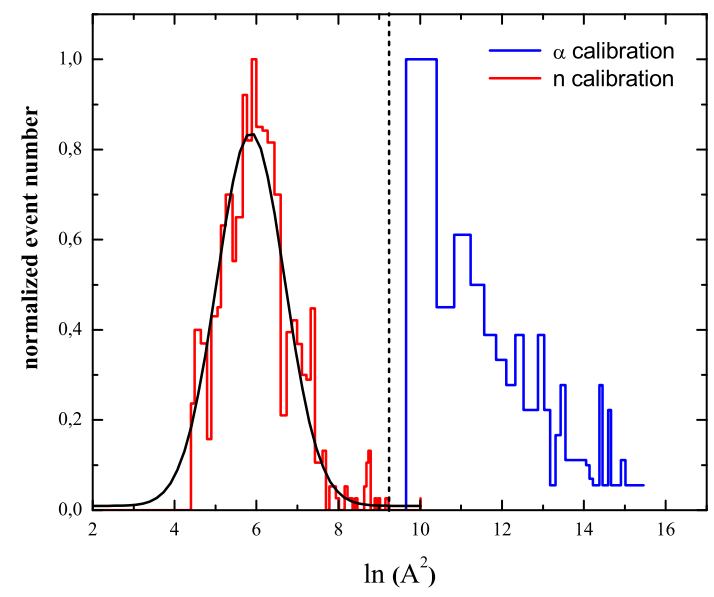

FIG. 2: A typical histogram of calibration neutron and $\alpha$ amplitudes, each population consisting of several hundred events. The vertical dashed line indicates the neutron discrimination cut at $\mathrm{A} \leq 100 \mathrm{mV}$, which includes all neutron calibration events.

The 14 low amplitude events of the run are consistent with the neutron calibrations, yielding $0.99 \pm 0.27$ (stat) evt/kgd. Corrected for detection and identification efficiencies, the difference between measurement and neutron background estimate is better than $0.3 \sigma$.

An upper limit on the number of WIMP events in the presence of the uncertain neutron background is estimated by applying the Feldman-Cousins method [16], based on observing 14 events with a background one standard deviation below the central value of the expected neutron background. This yields 4.3 events, and a resulting WIMP rate of $0.57 \mathrm{evt} / \mathrm{kgd}$ at $90 \%$ C.L.

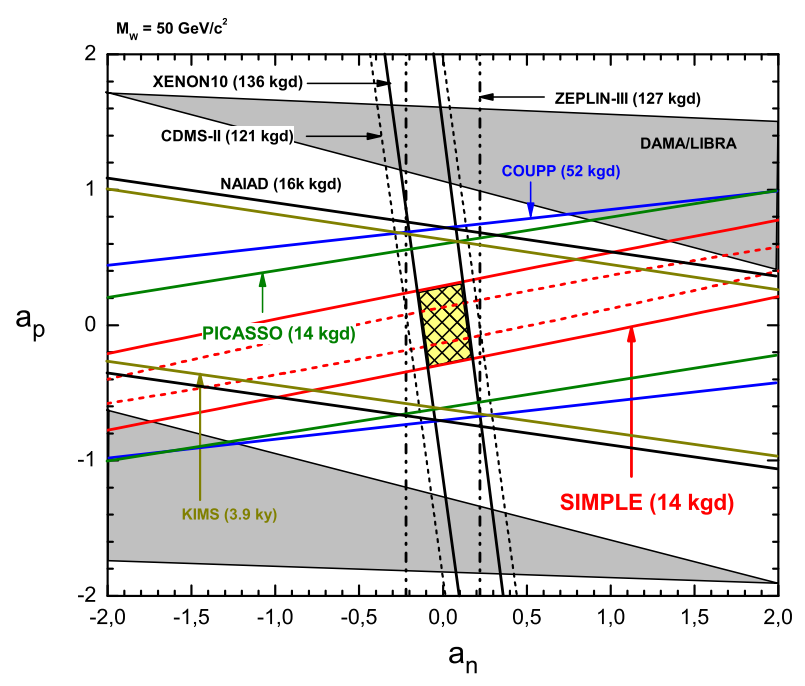

FIG. 3: $\mathrm{a}_{p}-\mathrm{a}_{n}$ for SIMPLE at $\mathrm{M}_{W}=50 \mathrm{GeV} / \mathrm{c}^{2}$, together with benchmark experiment results; the dashed contour represents a "0 evt" SIMPLE result for the same exposure. The allowed regions are defined by a band (single nuclei target) or ellipse (multinuclei target), with the external area excluded. The cross-hatched central area about $(0,0)$ indicates the region allowed by this result and XENON.

The impact of the result in the SD phase space at $\mathrm{M}_{W}$ $=50 \mathrm{GeV} / \mathrm{c}^{2}$ is shown in Fig. 3. The contour is calculated within a model-independent formulation [10], in which the region excluded by an experiment lies outside the indicated band, and the allowed region is defined by the intersection of the various bands. The calculations use a standard isothermal halo, bubble nucleation efficiency of $\left(1-\mathrm{E}_{t h r} / \mathrm{E}\right)$ [6], and WIMP scattering rate [17] with zero momentum transfer spin-dependent cross section $\sigma_{S D}$ for elastic scattering:

$$
\sigma_{S D} \sim G_{F}\left[a_{p}<S_{p}>+a_{n}<S_{n}>\right]^{2} \frac{J+1}{J}
$$

where $a_{p, n}$ are the WIMP-proton,neutron coupling strengths, $\left\langle S_{p, n}>\right.$ are the expectation values of the proton (neutron) group's spin, $\mathrm{G}_{F}$ is the Fermi coupling constant, and $\mathrm{J}$ is the total nuclear spin. The form factors of Ref. [17] have been used for all odd-A nuclei. The spin values of Strottman have been used for ${ }^{19} \mathrm{~F}$ [18]; for ${ }^{35} \mathrm{Cl}$ and ${ }^{37} \mathrm{Cl},\left\langle S_{p, n}>\right.$ are from Ref. [10], while for ${ }^{13} \mathrm{C}$ the $\left\langle S_{p, n}\right\rangle$ were estimated by using the odd group approximation. Use of the Divari et. al. spin values [19] for ${ }^{19} \mathrm{~F}$ would rotate the ellipse about the origin to a more horizontal position. We include only the $121 \mathrm{kgd}$ result of CDMS 2] since the more recent [20] model-independent result hasn't yet been published. The shaded area represents the allowed DAMA/LIBRA region [21], which is already excluded by other experiments. 
As indicated, the present result combined with XENON10 yields limits of $\left|a_{p}\right| \leq 0.32,\left|a_{n}\right| \leq 0.16$ on the SD sector of WIMP-nucleus interactions for $\mathrm{M}_{W}=50$ $\mathrm{GeV} / \mathrm{c}^{2}$, with $\sim 50 \%$ reduction in the allowed region of the phase space. $\mathrm{M}_{W}$ above or below this choice yield less restrictive limits [10].

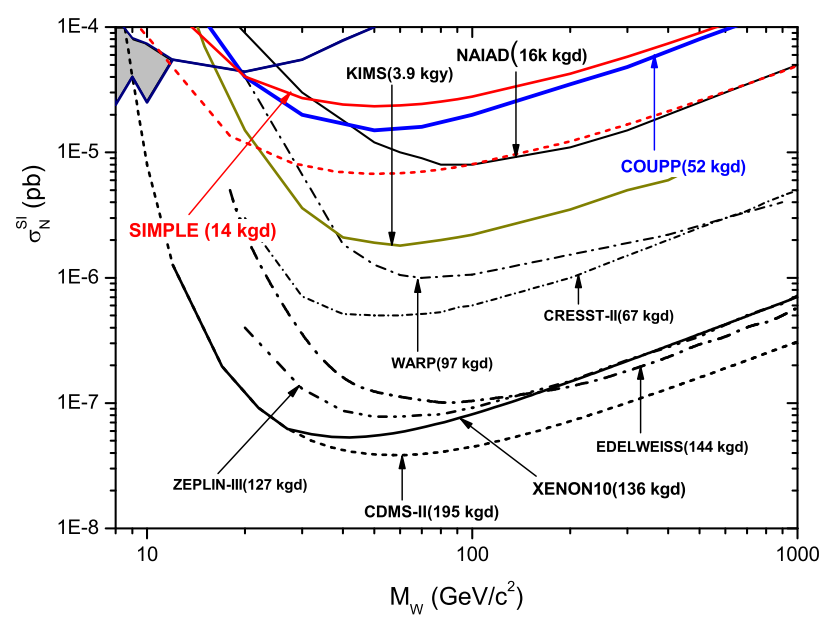

FIG. 4: Spin-independent contour for SIMPLE; the dashed contour represents a " 0 evt" SIMPLE result for the same measurement exposure. Also shown are several [22] of the leading spin-independent search results; the shaded area represents the recent result of CoGeNT 23].

For completeness, the impact of the result in the SI sector, calculated following the standard isothermal halo and WIMP elastic scattering rate of Ref. [17] using a Helm form factor, is shown in Fig. 4 in comparison with results from other leading search efforts 22]. The figure indicates a contour minimum of $2.3 \times 10^{-5} \mathrm{pb}$ at 45
$\mathrm{GeV} / \mathrm{c}^{2}$, slightly less restrictive than that of the $52 \mathrm{kgd}$ COUPP [24] exposure. The near-equivalence of the two, despite the $\sim 48 \times$ difference in sensitivity from coherence enhancement, most likely derives from the high radiopurity and $\alpha$-discrimination of the SIMPLE detectors.

In summary, a conservative analysis of $14.1 \mathrm{kgd}$ of data from the first phase run of SIMPLE yields new restrictions on the WIMP-proton parameter space of SD WIMP interactions which improves on those from both PICASSO and COUPP. These results represent the first stage of the ongoing $30 \mathrm{kgd}$ Phase II exposure permitted by current funding, and further demonstrates the competitiveness of the superheated liquid technique in the search for astroparticle dark matter in both spin -dependent and -independent sectors. To further approach the dashed contours of SIMPLE in Figs. 3 and 4 requires further reduction and/or elimination of the neutron background: improvements in the second stage of this measurement include a $10 \mathrm{~cm}$ increase in the wood pedestal beneath the waterpool, and installation of an additional $10 \mathrm{~cm}$ polyethylene beneath the detectors, which together are projected to reduce the overall neutron contribution by $\sim$ factor 5 . This is accompanied by additional $\alpha$ and neutron calibrations, and a refined $\alpha$ neutron discrimination analysis [25] towards understanding the differences between these results and those of PICASSO 26].

We thank Dr. F. Giuliani for numerous suggestions and advices, Dr. P. Loaiza for the radioassays of the site concrete and steel, Eng J. Albuquerque of CRIOLAB, Lda for numerous technical assistances during the measurement staging, and the Casoli's for their hospitality during our various residences near the LSBB. This work was supported in part by grant PDTC/FIS/83424/2006 of the Portuguese Foundation for Science and Technology (FCT), and by the Nuclear Physics Center of the University of Lisbon.
[1] J. Angle et. al., Phys. Rev. Lett. 100, 021303 (2008).

[2] Z. Ahmed et. al., Phys. Rev. Lett. 102, 011301 (2009).

[3] V.N. Lebedenko et. al., Phys. Rev. D80, 052010 (2009).

[4] S. Archambault et. al., Phys. Lett. B682, 185 (2009).

[5] H.S. Lee et. al., Phys. Rev. Lett. 99, 091301 (2007).

[6] T. Morlat et. al., Astrop. Phys. 30, 159 (2008).

[7] TA Girard et. al., Phys. Lett. B621, 233 (2005).

[8] M. Felizardo et. al., Nucl. Instrum. Meth. A589, 72 (2008).

[9] F. Seitz, Phys. Fluids 1, 1 (1958).

[10] F. Giuliani and TA Girard, Phys. Rev. D71, 123503 (2005).

[11] G. Waysand et. al., Nucl. Instrum. Meth. A444, 336 (2000).

[12] J. Amaré et. al., Journ. Phys.: Conf. Series 39, 151 (2006).

[13] V. Chazal et. al., Astrop. Phys. 9, 163 (1998).

[14] H. Wulandari et. al., Astrop. Phys. 22, 313 (2004).
[15] NCRP Report 97, Bethesda MD (1988).

[16] G.J. Feldman and R.D. Cousins, Phys. Rev. D57, 3873(1998).

[17] J.D. Lewin and P.F. Smith, Astrop. Phys. 6, 87(1996).

[18] A.F. Pacheco and D. Strottman, Phys. Rev. D40, 2131 (1989).

[19] M.T. Divari et. al., Phys. Rev. C61, 054612 (2000).

[20] Z. Ahmed et. al., Science 327, 1619 (2010).

[21] C. Savage et. al., JCAP 09, 036 (2009).

[22] The PICASSO impact is not indicated since their contour hasn't yet been presented.

[23] C.E. Aalseth, arXiv:1002.4703v2 [astro-ph.CO].

[24] E. Behnke et. al., Science 319, 933 (2008).

[25] Yu.N. Martynyuk and N.S. Smirnova, Sov. Phys. Acoust. 374, 376 (1991).

[26] F. Aubin et. al., New Journ. Phys. 10, 103017 (2008). 